\title{
Prospects for direct searches for light Higgs bosons at the ILC with $250 \mathrm{GeV}$
}

\author{
P. Drechsel ${ }^{1}$, G. Moortgat-Pick ${ }^{1,2, a}$, G. Weiglein ${ }^{1, b}$ \\ ${ }^{1}$ DESY, Notkestraße 85, 22607 Hamburg, Germany \\ ${ }^{2}$ II. Institut für Theoretische Physik, Universität Hamburg, Luruper Chaussee 149, 22761 Hamburg, Germany
}

Received: 14 May 2020 / Accepted: 6 September 2020 / Published online: 7 October 2020

(C) The Author(s) 2020

\begin{abstract}
The particle discovered in the Higgs boson searches at the LHC with a mass of about $125 \mathrm{GeV}$ is compatible within the present uncertainties with the Higgs boson predicted in the Standard Model (SM), but it could also be identified with one of the neutral Higgs bosons in a variety of beyond the SM (BSM) theories with an extended Higgs sector. The possibility that an additional Higgs boson (or even more than one) could be lighter than the state that has been detected at $125 \mathrm{GeV}$ occurs generically in many BSM models and has some support from slight excesses that were observed above the background expectations in Higgs searches at LEP and at the LHC. The couplings between additional Higgs fields and the electroweak gauge bosons in BSM theories could be probed by model-independent Higgs searches at lepton colliders. We present a generator-level extrapolation of the limits obtained at LEP to the case of a future $e^{+} e^{-}$ collider, both for the search where the light Higgs boson decays into a pair of bottom quarks and for the decay-modeindependent search utilising the recoil method. We find that at the ILC with a centre-of-mass energy of $250 \mathrm{GeV}$, an integrated luminosity of $500 \mathrm{fb}^{-1}$ and polarised beams, the sensitivity to a light Higgs boson with reduced couplings to gauge bosons is improved by more than an order of magnitude compared to the LEP limits and goes much beyond the projected indirect sensitivity of the HL-LHC with $3000 \mathrm{fb}^{-1}$ from the rate measurements of the detected state at $125 \mathrm{GeV}$.
\end{abstract}

\section{Introduction}

The properties of the Higgs boson that was discovered in 2012 are in agreement with the predictions of the Standard

Former address: P. Drechsel.

a e-mail: gudrid.moortgat-pick@ desy.de (corresponding author)

be-mail: georg.weiglein@desy.de
Model (SM) within the current experimental accuracy, but they are also compatible with a wide variety of extensions of or alternatives to the SM. Extended Higgs sectors predict the existence of additional Higgs bosons, which could be heavier but also lighter than the observed state at $125 \mathrm{GeV}$. The coupling of the SM Higgs boson to the gauge bosons $W$ and $Z$ is such that terms with a bad high-energy behaviour in longitudinal vector-boson scattering exactly cancel with each other. As a consequence, in an extended Higgs sector comprising the SM-like state at $125 \mathrm{GeV}$ and additional Higgs bosons the couplings of the additional neutral Higgs bosons to $W$ and $Z, g_{\phi_{i} V V}$, are expected to be small. This implies that additional heavy neutral Higgs bosons may not be detectable via the search channels $\phi_{i} \rightarrow Z Z, W^{+} W^{-}$and that for an additional light Higgs boson (or more than one) $g_{\phi V V}$ may be so small that such a light Higgs boson would have escaped the limits from the Higgs searches at LEP [1,2] (see also Ref. [3]) and the Tevatron [4].

The case of an extended Higgs sector containing a SMlike state that can be identified with the observed Higgs signal and further Higgs bosons of which at least one is lighter than $125 \mathrm{GeV}$ can be realised, for instance, in a general two-Higgsdoublet model ${ }^{1}$ (2HDM), and it occurs generically in extensions with a light singlet such as the next-to-minimal supersymmetric extension of the SM (NMSSM), see e.g. Refs. [79], or the N2HDM, a 2HDM with an additional real Higgs singlet, see e.g. Ref. [10]. It should be noted that extensions of the SM with a singlet-dominated state in the mass range around or just below $100 \mathrm{GeV}$ are also of interest in view of the observed local excesses around $96 \mathrm{GeV}$ at the $2-3 \sigma$ level in both the searches at LEP in the $e^{+} e^{-} \rightarrow Z \phi, \phi \rightarrow b \bar{b}$ channel [1,2] and at CMS in the $\phi \rightarrow \gamma \gamma$ searches [11] (the CMS result is compatible [12] with the results of the corresponding searches at ATLAS [13]). Possible interpreta-

\footnotetext{
${ }_{1}$ See e.g. Refs. $[5,6]$ for recent discussions of the viability of such a scenario in the minimal supersymmetric extension of the SM (MSSM).
} 
tions have been discussed e.g. in the NMSSM $[9,14,15]$, an inflation-inspired $\mu$ NMSSM $[16,17]$ and the N2HDM [10].

Specifically, the neutral Higgs bosons $\phi_{i}$ of extended Higgs sectors consisting of any number of doublets and singlets fulfill the sum rule at lowest order that the squared couplings to gauge bosons of all $\phi_{i}$ add up to the squared coupling of the SM Higgs boson to gauge bosons,

$\sum_{i}\left(g_{\phi_{i} V V}\right)^{2}=\left(g_{H V V}^{\mathrm{SM}}\right)^{2}, \quad$ where $V \in\{W, Z\}$.

Accordingly, this sum rule is valid for a wide class of models, including all the examples of beyond the Standard Model (BSM) theories with an extended Higgs sector mentioned above. The sum rule receives corrections at the loop level, but these amount to effects that are typically at the per cent level. This pattern of extended Higgs sectors implies on the one hand that the couplings of the observed state at $125 \mathrm{GeV}$ should be measured with the highest possible precision in order to maximise the sensitivity for establishing a deviation from the SM values, and on the other hand it provides a strong motivation to search for additional Higgs bosons with couplings to gauge bosons that are significantly suppressed compared to the case of a SM-like Higgs boson with the same mass. As far as the search for light additional Higgs bosons is concerned, the mass region between about $60 \mathrm{GeV}$ (since for $2 m_{\phi}>125 \mathrm{GeV}$ the decay of the Higgs boson at $125 \mathrm{GeV}, h(125)$, into a pair of the additional Higgs bosons, $h(125) \rightarrow \phi \phi$, is kinematically closed) and $100 \mathrm{GeV}$ appears to be particularly promising, as this mass range is only mildly constrained by the existing limits from the Higgs searches at LEP and the $\phi \rightarrow \gamma \gamma$ searches at the LHC (and as discussed above, in both types of these searches an interesting excess above the background expectation has been reported).

In the current paper we study the sensitivity of searches at the ILC in its first stage of $\sqrt{s}=250 \mathrm{GeV}$ centre-of-mass energy for light additional Higgs bosons with masses below the one of the observed signal at $125 \mathrm{GeV}$. Due to the clean environment and consequently a very favourable signal-tobackground ratio for the prospective integrated luminosity, the low beamstahlung, the precise knowledge of the beam energy and the availability of polarised beams, the ILC has a high physics potential in the direct search for such light additional Higgs bosons. We perform a generator-level extrapolation of the limits obtained at LEP to the ILC case, both for the search for the $\phi \rightarrow b \bar{b}$ final state and for the decaymode independent recoil technique (the latter was pioneered at LEP by the OPAL collaboration [3] and forms the basis for the total cross-section measurement for $Z h(125)$ production at future $e^{+} e^{-}$colliders).

The two main production processes for a neutral Higgs boson at the ILC are Higgs-strahlung $\left(e^{+} e^{-} \rightarrow(H / \phi) Z\right)$, dominant at lower masses and lower collider energies, and
$W W$-fusion $\left(e^{+} e^{-} \rightarrow(H / \Phi) v \bar{v}\right)$, dominant at higher Higgs masses and higher collider energies. In the current study, we therefore focus on the Higgs-strahlung process, for which we investigate the $\phi \rightarrow b \bar{b}$ channel as well as the recoil method, where only the leptonic $Z$-boson decays are utilised for the reconstruction of the final state (in our study we have concentrated on the $Z \rightarrow \mu^{+} \mu^{-}$decay). We first validate our approach with the LEP results and then perform an extrapolation to the case of the ILC. The present paper builds up on a preliminary study that was carried out in Ref. [18], see also Ref. [19].

The paper is organised as follows. The adopted statistical method is described in Sect. 2. In Sect. 3 the method is validated with the results obtained at LEP, and in Sect. 4 the sensitivity of the ILC searches for light Higgs bosons is discussed. Our conclusions are given in Sect. 5.

\section{Description of the applied methods}

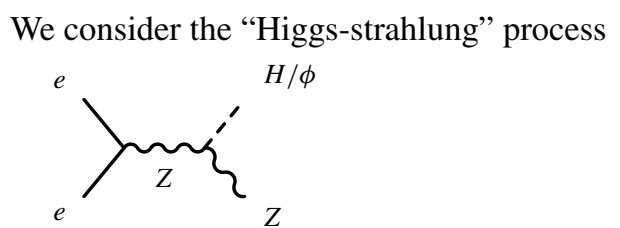

where for our generator-level analysis we focus on the $Z$ boson decay into muons, $Z \rightarrow \mu^{+} \mu^{-}$, and treat the cases where either the decay of the scalar $H / \phi \rightarrow b \bar{b}$ is reconstructed,

$e^{+}+e^{-} \rightarrow Z+(H / \phi) \rightarrow b+\bar{b}+\mu^{-}+\mu^{+}$,

or only the information from the leptonic $Z$-boson decay is used. For the scalar either the SM Higgs boson $H$ is considered or a light Higgs boson $\phi$ with a reduced coupling to gauge bosons compared to the case of a SM-like Higgs boson with the same mass.

Concerning the statistical treatment we follow the prescription that was outlined for $S_{95}$ in Refs. [1,20], using a simplified approach, see also [21]. We have generated event samples corresponding to the two hypotheses "all events are generated by the background only" or "all events are generated by the background plus a hypothetical signal". This has been done by comparing the events for the full $e^{+} e^{-} \rightarrow 4$ fermion process for the considered mass value of the assumed signal, taking into account all interference contributions, with the events for the full $e^{+} e^{-} \rightarrow 4$ fermion process for the case where the mass value of the assumed signal is beyond the kinematic reach of the collider. The quantity

$S_{95}=\frac{\hat{\sigma}}{\sigma_{\text {ref }}}=\frac{\hat{n}}{n}$ 
gives an upper limit $\hat{\sigma}$ on a cross section that is compatible with the "background only" hypothesis at the 95\% confidence level, normalised to a reference cross section $\sigma_{\text {ref }}$, or equivalently on the ratio of the allowed signal rate $\hat{n}$, normalised to the reference signal rate $n$. As reference process we use in Eq. (3) the case of the SM Higgs boson at the considered mass value. Accordingly, Eq. (4) can be interpreted as the ratio between the squared couplings of the scalars $\phi$ and $H$ to the $Z$-boson,

$S_{95} \hat{=}\left|\frac{g_{\phi Z Z}^{2}}{g_{H Z Z}^{2}}\right|$,

for each mass value.

We have generated event samples for the process

$e^{+}+e^{-} \rightarrow b+\bar{b}+\mu^{-}+\mu^{+}$

with the Monte Carlo generator Whi zard-2 . 4 . 1 [22,23], which we apply for both signal processes and their reference values (with and without reconstruction of the $H / \phi \rightarrow b \bar{b}$ decay) as well as for the corresponding backgrounds (a simple cut has been applied to control backgrounds with photon radiation). Since we generate the full $e^{+} e^{-} \rightarrow 4$ fermion process, the corresponding signal and background events are not generated separately. The obtained events are classified by either the invariant mass of the quark system or the recoil mass of the lepton system for this analysis. The signal rate in the $i$-th bin (we use a width of $1 \mathrm{GeV}$ for the mass bins) is determined by the difference of the event rates for the background plus signal $\left(s b_{i}\right)$ and the background only hypotheses $\left(b_{i}\right)$, and the signal rate $s_{i}$ is obtained by $s b_{i}-b_{i}$. Negative rates for signal events are cut, $s_{i}=\max \left(0, s b_{i}-b_{i}\right)$.

We assume that the number of potential signal events $d_{i}$ in each of the $i$ bins are distributed according to a Poisson distribution with the expected values $b_{i}$ for the "background only" and $s_{i}+b_{i}$ for the "background plus signal" hypotheses, respectively. The test statistic

$Q=\frac{L_{s+b}}{L_{b}}=e^{-n} \prod_{\text {bins } i} \frac{\left(s_{i}+b_{i}\right)^{d_{i}}}{\left(b_{i}\right)^{d_{i}}}$

orders the outcome of test experiments according to their "signal likeness". While the expectation values of the Poisson distributions have to be determined a priori, the numbers of potential signal events $d_{i}$ have to be determined experimentally. For this work we consider only simulated events, and thus $d_{i}=s_{i}$. The logarithm of the test-statistic yields the weights for the number of potential signal events per bin as

$w_{i}=\log \left(1+\frac{s_{i}}{b_{i}}\right)$
This definition requires the presence of at least one background event per considered bin. In order to accommodate this, we choose the luminosity for the simulation large enough that at least one event can be found in each bin, i.e. $b_{i} \geq 1$, and scale the luminosity afterwards with a factor $c$ to the desired value. The weights $w_{i}$ are not affected by the scaling procedure.

With the weight factors of Eq. (8), the scaling factor $S_{95}$ given in Eq. (4) can be expressed [20] as

$S_{95}=\frac{\hat{n}}{n}=\frac{K \cdot \sigma_{s b}}{\langle X\rangle_{s}}=\frac{1.96 \cdot \sigma_{s b}}{\langle X\rangle_{s}}$,

where $K$ denotes the number of standard deviations for the required significance: for $S_{95}$ the value of $K=1.96$ is used, corresponding to a confidence level (C.L.) of 5\% for the signal hypothesis. The variance $\sigma_{s b}$ is given by $\sigma_{s b}^{2}=\sum_{\text {bins } i} w_{i}^{2}\left(s_{i}+b_{i}\right)$, and $\langle X\rangle_{s}=\sum_{\text {bins } i} w_{i} s_{i}$.

\section{Validation of the methods with results from LEP I and LEP II}

In order to validate the described method we apply it in a first step to the search in the $\phi \rightarrow b \bar{b}$ channel and compare with the results obtained by the LEP combination [1,2]. Furthermore, in a second step, we validate our approach for the case of the search via the recoil method by comparing with the results obtained by the OPAL collaboration in their Higgs analysis employing the recoil method [3].

We simulated the process $e^{+}+e^{-} \rightarrow b+\bar{b}+\mu^{+}+\mu^{-}$with the SM implementation of Whi zard 2.4.1 [22,23]. This process contains for the signal events the Higgs-strahlung process where the produced Higgs boson decays into $b \bar{b}$ and the $Z$ boson decays into $\mu^{+} \mu^{-}$. As described above, for simplicity we restrict to the $b \bar{b} \mu^{+} \mu^{-}$final state, i.e. we do not perform a separate analysis for the $b \bar{b} e^{+} e^{-}$final state and for our analysis via the recoil method we also do not simulate additional decay modes of the Higgs boson. As explained above, our "background only" and "background plus signal" hypotheses are generated by appropriately adapting the mass of the implemented SM-like Higgs boson, i.e. for the "background only" hypothesis the mass $m_{\phi}$ of the scalar is chosen beyond the kinematic accessibility of the LEP experiments.

For LEP 1 we consider the integrated luminosity of the four experiments at the centre-of-mass energy of $91.2 \mathrm{GeV}$. For LEP 2 we use for the comparison the integrated luminosity that the four LEP experiments recorded in a particular year and assign it to the highest energy that was achieved during that year, with the exception of the last year of LEP running. For the latter we use the energy of $206 \mathrm{GeV}$ [24], where the bulk of the luminosity was recorded during that year. The luminosity per experiment that we have used in 
Table 1 The integrated luminosities per experiment, $\int \mathrm{d} t \mathcal{L}$, at the different stages of the LEP centre-of-mass energies, $\sqrt{s}$, that are used for the comparison with the LEP results (see text)

\begin{tabular}{lllllll}
\hline$\sqrt{s} / \mathrm{GeV}$ & 91.2 & 172 & 184 & 189 & 202 & 206 \\
$\int \mathrm{d} t \mathcal{L} / \mathrm{pb}^{-1}$ & 208.44 & 24.7 & 73.4 & 199.7 & 253 & 233.4 \\
\hline
\end{tabular}

our comparison is given for the different LEP energy stages in Table 1 . The number of events that we actually generated for our simulation is in fact 400 times higher than the LEP luminosity in order to facilitate the determinaton of the weight factors $w_{i}$, see Eq. (8), and to reduce the statistical error. The results were then scaled down to the appropriate luminosities as described above.

In our generator-level analysis we do not take into account hadronisation effects of the $b$-quarks and we also do not simulate detector effects. This simplification leads to an overoptimistic estimate for the signal efficiencies and thus for the observed signal rates. Since for the searches making use of the $\phi \rightarrow b \bar{b}$ final state the signal efficiencies have not explicitly been given for the LEP experiments [21], we introduce a scale factor multiplying the luminosity that we use for the comparison with the LEP 1 results and we also allow for such a factor for the comparison of the LEP 2 results. While our over-optimistic treatment of the signal efficiencies implies the need to scale down our effective luminosities, we also need to appropriately scale them up to account for the fact that we have simulated only events for the $Z \rightarrow \mu^{+} \mu^{-}$final state, whereas the LEP analyses also incorporate the other decay modes of the $Z$ boson. Both effects can be combined into a single scale factor. We introduce such a factor, $c_{\mathrm{bb}}$, for the $\phi \rightarrow b \bar{b}$ analyses both at LEP 1 and LEP 2 . The corresponding factors for the recoil method analyses at LEP 1 and LEP 2 are denoted as $c_{\text {recoil }}$. Specifically, we obtain the effective luminosities $\mathcal{L}_{\text {eff }}$ for our comparison as

$\mathcal{L}_{\text {eff }}^{\text {bb }}=c_{\text {bb }} \cdot \mathcal{L}, \quad \mathcal{L}_{\text {eff }}^{\text {recoil }}=c_{\text {recoil }} \epsilon \cdot \mathcal{L}$,

where $\mathcal{L}$ is the luminosity derived from the values given in Table 1 . The scaling factors $c_{\mathrm{bb}}, c_{\text {recoil }}$ depend on the parameters of the experiment and the analysis. We have determined those factors from a comparison with the LEP-combined results for $S_{95}$ in the case of the $\phi \rightarrow b \bar{b}$ analyses and with the OPAL results for the case of the recoil method analyses. The four approximate scaling factors, rounded to integer values, that we have obtained in this way are listed in Table 2.

Obviously such a simple scaling factor can only roughly approximate an actual experimental analysis. For the case of the $\phi \rightarrow b \bar{b}$ analysis the scaling factor for incorporating besides the $Z \rightarrow \mu^{+} \mu^{-}$final state also the events for the other $Z$ decay modes would roughly correspond to an increase of the effective luminosity by a factor of 30 for an
Table 2 Luminosity scaling factors $c_{\mathrm{bb}}$ and $c_{\text {recoil }}$ derived via comparison with the $S_{95}$ results for the LEP $1(91.2 \mathrm{GeV})$ and LEP 2 ( $>91.2 \mathrm{GeV}$ ) energy stages from the LEP combination for the analysis using the $\phi \rightarrow b \bar{b}$ final state and from the OPAL results for the recoil method analysis

\begin{tabular}{lll}
\hline$\sqrt{s} / \mathrm{GeV}$ & 91.2 & $>91.2$ \\
\hline$\phi \rightarrow b \bar{b}$ & $c_{\mathrm{bb}}=12$ & $c_{\mathrm{bb}}=4$ \\
Recoil method & $c_{\text {recoil }}=4$ & $c_{\text {recoil }}=1$ \\
\hline
\end{tabular}

ideal detector. Assuming for simplicity a median signal efficiency of $50 \%$ for the signal rates leads to the conclusion that the coefficients $c_{\text {bb }}$ in Table 2 should not be larger than $\approx 15$. The comparison with the listed results for $c_{\mathrm{bb}}$ shows that the value that we obtained for the LEP 1 case $(91.2 \mathrm{GeV})$ is rather close to the expectation for this idealised case. For the LEP 2 case $(>91.2 \mathrm{GeV})$, on the other hand, the detector effects play a larger role, and our generator-level extrapolation corresponds to a more optimistic estimate in comparison with the analysis incorporating a realistic treatment of the backgrounds and the experimental efficiencies. Table 2 shows that for the recoil method the required correction factors $c_{\text {recoil }}$ are smaller. This is expected from the facts that the OPAL analysis only made use of the decay modes $Z \rightarrow \mu^{+} \mu^{-}, e^{+} e^{-}$, that for this analysis explicit signal efficiencies $\epsilon$ have been published, which we have taken into account in Eq. (10), and that generally the detector effects are expected to be less important for the recoil analysis as it only relies on a leptonic final state.

\subsection{Comparison with LEP data using the $H / \phi$ decay mode}

In order to compare our analysis for the search using the $H / \phi$ decay mode with the LEP data, we use the event samples for the process $e^{+}+e^{-} \rightarrow b+\bar{b}+\mu^{+}+\mu^{-}$and employ the information from the reconstruction of the $b \bar{b}$ system for the identification of the samples for "background only", $b_{i}$, and "background plus signal", $b_{i}+s_{i}$. The information from the $\mu^{+} \mu^{-}$system is only used for validating that the signal events are compatible with the production of a Higgs boson together with a $Z$ boson. A challenging region for this analysis is where the scalar mass $m_{\phi}$ is close to $M_{Z}$, since the presence of a large number of background events close to the $Z$-boson mass $M_{Z}$ weakens the limit on $S_{95}$; in this region we fit the expected background events to emulate a more detailed analysis: we extrapolate the expected background events in an interval around $M_{Z}$ and fit these points with a third-order polynomial to obtain the number of events in the bins around $M_{Z}$ as the value of the fit function at the central mass of each bin.

The result using our method for approximately reconstructing the expected LEP limit is shown in Fig. 1 in com- 


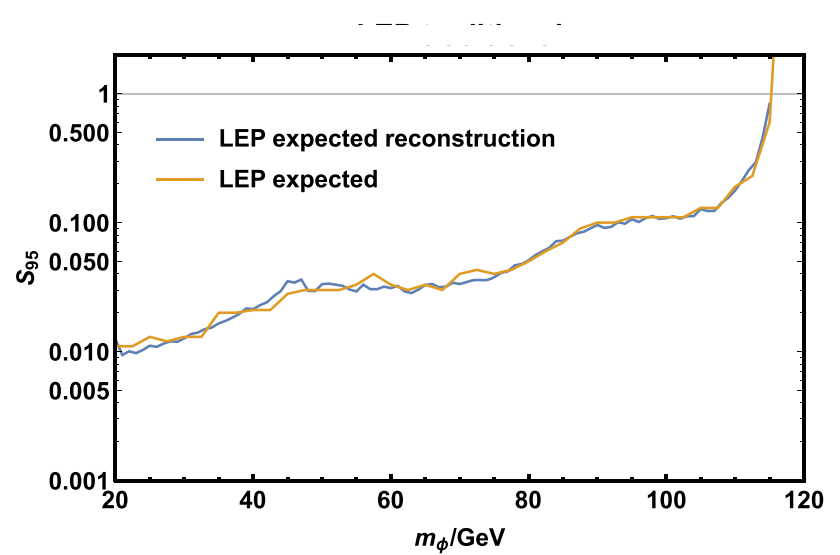

Fig. 1 The expected limit from LEP for the analyses using the $\phi \rightarrow$ $b \bar{b}$ decay information is compared with our method for approximately reconstructing the expected limit for $S_{95}$ from the process $e^{+} e^{-} \rightarrow$ $Z(H / \phi) \rightarrow \mu^{+} \mu^{-} b \bar{b}$. Our approximate result makes use of the two scaling factors $c_{\mathrm{bb}}$ given in Table 2

parison with the expected limit that was published by the LEP collaborations. As explained above, our method makes use of the two scaling factors $c_{\mathrm{bb}}$ given in Table 2. The comparison in Fig. 1 shows that with those two factors as input our method reproduces very well not only the normalisation of the expected LEP limit but also its shape as a function of $m_{\phi}$.

\subsection{Comparison with OPAL data using the recoil method}

The OPAL collaboration also used the recoil method for analysing the data, i.e. exploiting the recoil of $H, \phi$ from the $Z$-boson and analysing the $Z$ decay only. This method has the great advantage of being completely independent of the $H, \phi$ decay modes.

The OPAL analysis used the decay modes $Z \rightarrow \mu^{+} \mu^{-}$, $e^{+} e^{-}$[3]. We restrict our analysis to the decay mode $Z \rightarrow$ $\mu^{+} \mu^{-}$only, but extrapolate in a second step the luminosity taken by OPAL to the full LEP luminosity, see Table 1 . We include only bins close to $M_{Z}$ (interval [ $\left.84 \mathrm{GeV}, 98 \mathrm{GeV}\right]$ ), so that the weighted mean of the central masses of the bins is in a small interval around $M_{Z}$

$$
\frac{\sum_{i=1}^{N} d_{i} m_{i}}{\sum_{j=1}^{N} d_{j}} \in[91.1 \mathrm{GeV}, 91.3 \mathrm{GeV}]
$$

with the number of events $d_{i}$ in the $i$ th of $N$ bins with the central mass $m_{i}$.

The events from the $\mu$-lepton pairs are ordered by their total energy $E_{i}$ into bins, and for each bin we calculated the respective recoil mass $m_{i}^{(\mathrm{rec})}$,

$m_{i}^{(\mathrm{rec})}=\sqrt{s+M_{Z}^{2}-2 E_{i} \sqrt{s}}$,

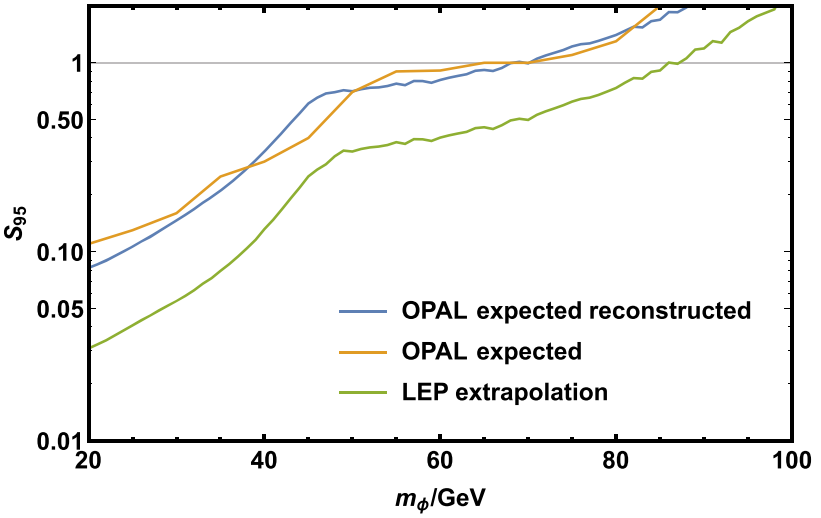

Fig. 2 The expected limit from OPAL based on the recoil method is compared with our method for approximately reconstructing the expected limit for $S_{95}$, which makes use of the two scaling factors $c_{\text {recoil }}$ given in Table 2. Also shown is our result where the luminosity has been extrapolated to the full LEP luminosity

to obtain the event rates for background, $b_{i} \equiv b_{i}\left(m_{i}^{(\mathrm{rec})}\right)$, and signal, $s_{i} \equiv s_{i}\left(m_{i}^{(\mathrm{rec})}\right)$.

Since the OPAL collaboration published the signal efficiency of their analysis in Ref. [3], we could make use of this information to estimate a mean signal efficiency of $\varepsilon=30 \%$ for our approach and obtained in this way the effective luminosity $\mathcal{L}_{\text {eff }}$ according to Eq. (10). The obtained values for the two scaling factors $c_{\text {recoil }}(\sqrt{s})$ are given in Table 2.

Our approximate reconstruction of the expected limit for $S_{95}$ from the recoil method analysis is compared with the expected limit published by the OPAL collaboration in Fig. 2 , showing overall a good agreement. We furthermore display our result where the luminosity has been extrapolated to the full LEP luminosity.

\section{Discovery potential at the ILC for a light Higgs boson}

After having validated our method with the existing results from the analyses at LEP data, we are now in a position to apply this method in order to derive the expected limits for the ILC at $\sqrt{s}=250 \mathrm{GeV}$ for the two types of analyses making use of the $\phi \rightarrow b \bar{b}$ decay information and employing the recoil method. For our ILC analysis we use the two scaling factors $c_{\mathrm{bb}}$ and $c_{\text {recoil }}$ that we determined for LEP 2 as given in Table 2. Concerning the ILC we assume a beam polarisation of $P_{e^{-}}=-80 \%$ for the electron beam and $P_{e^{+}}=+30 \%$ for the positron beam, corresponding to the baseline design [25]. At the ILC with $\sqrt{s}=250 \mathrm{GeV}$ a total luminosity of $\mathcal{L}=2000 \mathrm{fb}^{-1}$ is expected to be collected within 15 years [26]. For our study, however, we exploit only the polarisation configuration $(-80 \%,+30 \%)$ and assume the rather modest luminosity of $500 \mathrm{fb}^{-1}$. 


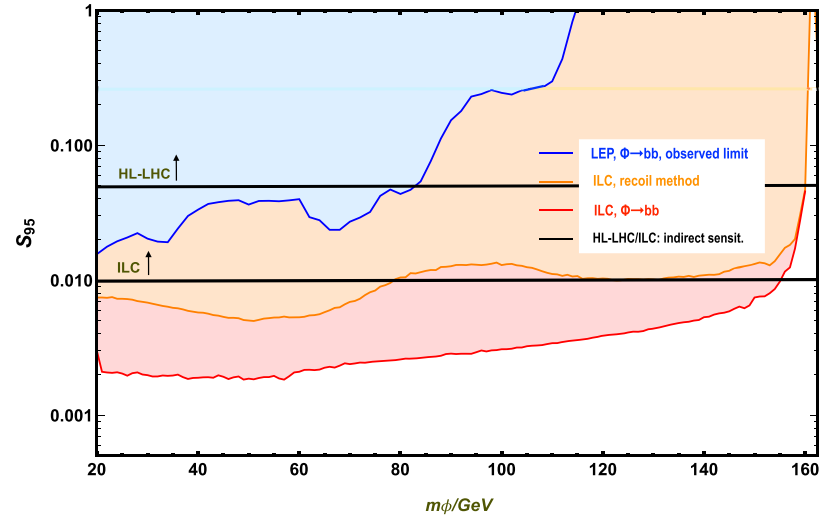

Fig. 3 Projections for the expected limits on $S_{95}$ at the ILC [19] with $\sqrt{s}=250 \mathrm{GeV}, \mathcal{L}=500 \mathrm{fb}^{-1}$ and polarised beams $\left(P_{e^{-}}=-80 \%\right.$ and $P_{e^{+}}=+30 \%$ ) for the analysis using the decay $\phi \rightarrow b \bar{b}$ and for the recoil method analysis, based only on the recoil from the $Z$ boson that is reconstructed from the $Z \rightarrow \mu^{+} \mu^{-}$decay. The impact of the Higgs signal at $125 \mathrm{GeV}$ is not shown. The ILC projections are compared with the observed limit at LEP from the $\phi \rightarrow b \bar{b}$ searches [1]. The solid horizontal lines denote the indirect sensitivities at the HL-LHC and the ILC with $\sqrt{s}=250 \mathrm{GeV}$ from the projection for the accuracies on the couplings of the observed Higgs boson at $125 \mathrm{GeV}$ [27] (see text)

In Fig. 3 we show our results for the $S_{95}$ projections at the ILC for the analysis making use of the decay $\phi \rightarrow b \bar{b}$ decay and and for the recoil method, where only the information from $Z \rightarrow \mu^{+} \mu^{-}$has been used [19] (the expected limits do not take into account the impact of the Higgs signal at $125 \mathrm{GeV})$. These results are compared with the observed limit from the $\phi \rightarrow b \bar{b}$ search at LEP [1]. As one can see from Fig. 3, the projected ILC limits yield an improvement of more than a factor of 10 in $S_{95}$ for the mass range between about $60 \mathrm{GeV}$ and $100 \mathrm{GeV}$ compared to the existing limits from LEP already for the moderate luminosity of $\mathcal{L}=500 \mathrm{fb}^{-1}$ at $\sqrt{s}=250 \mathrm{GeV}^{2}$

For comparison, in Fig. 3 also the indirect sensitivity at the HL-LHC from the projection for the accuracies on the couplings of the observed Higgs boson at $125 \mathrm{GeV}$ [27] is indicated. It corresponds to the area of the plot above the solid horizontal black line labelled as "HL-LHC". The rate measurements of the state at $125 \mathrm{GeV}$ provide an indirect sensitivity to the squared coupling $\left(g_{\phi Z Z}\right)^{2}$ of an additional light (or heavy) Higgs boson $\phi$ under the assumption that the sum rule of Eq. (1) is valid. The projected accuracy on the coupling of the Higgs boson at $125 \mathrm{GeV}, h(125)$, to $Z$ bosons, $\Delta g_{h(125) Z Z}$, translates under this assumption into an upper bound on the squared coupling of the light Higgs boson according to

\footnotetext{
${ }^{2}$ For one of the two types of analyses addressed in our paper, namely the analysis using the recoil method, meanwhile an ILC study has been performed with full ILD detector simulation [28,29]. The results obtained in Refs. $[28,29]$ are in good qualitative agreement (in view of the more complete treatment of backgrounds arising from events involving photons) with the curve in Fig. 3 showing our projection for the ILC based on the recoil method.
}

$\frac{\left(g_{\phi Z Z}\right)^{2}}{\left(g_{H Z Z}^{\mathrm{SM}}\right)^{2}} \leq 1-\left(1-\left|\Delta g_{h(125) Z Z}\right|\right)^{2}$

up to higher-order corrections to the sum rule. The indirect sensitivity achievable at the HL-LHC that is indicated in Fig. 3 is based on the projection for the $2 \sigma$ accuracy of the $g_{h(125) Z Z}$ coupling from Table 4 of Ref. [27], where an integrated luminosity of $3000 \mathrm{fb}^{-1}$ was assumed. While the indirect sensitivity at the HL-LHC exceeds the observed limit from LEP for $m_{\phi} \gtrsim 85 \mathrm{GeV}$, Fig. 3 clearly demonstrates that the sensitivity of the direct search at the ILC will much surpass the indirect sensitivity at the HL-LHC even for the ultimate accuracy reachable at the HL-LHC.

The indirect sensitivity of the ILC with $\sqrt{s}=250 \mathrm{GeV}$ from the rate measurements of the state at $125 \mathrm{GeV}$, where as for the HL-LHC we have used the projections listed in Table 4 of Ref. [27], is indicated in Fig. 3 as the area above the solid horizontal black line that is labelled as "ILC". Fig. 3 shows that this indirect sensitivity at the ILC would be similar to the direct reach via the recoil method, which could provide important complementary information for determining the nature of a possible excess in the direct searches for additional light Higgs bosons at the ILC. It should be noted that the sensitivity of the direct ILC searches for additional Higgs bosons making use of the $\phi \rightarrow b \bar{b}$ decay mode will significantly improve even on the indirect sensitivity of the ILC based on its high-precision measurement of the coupling of $h(125)$ to $Z$ bosons.

\section{Conclusions}

In this paper we have pointed out that the ILC at $\sqrt{s}=$ $250 \mathrm{GeV}$ has a large physics potential in the direct search for additional light Higgs bosons. Such a light Higgs boson would be expected to have a heavily suppressed coupling to gauge bosons as compared to a SM-like Higgs boson at the same mass, which could therefore be below the existing limits from LEP. Via those direct searches the ILC would probe favoured parameter regions of various extensions of the SM, which have recently received considerable attention also in view of the excess over the background expectation that has been reported for the $\phi \rightarrow \gamma \gamma$ searches at CMS in the vicinity of a long-standing excess in the LEP Higgs searches.

We have performed a generator-level study for searches via the Higgs-strahlung process at a future $e^{+} e^{-}$collider for both the analysis type using the $\phi \rightarrow b \bar{b}$ decay and for the decay-mode independent search via the recoil method. In a first step we have validated our approach with the existing results for the search in the $\phi \rightarrow b \bar{b}$ channel, from the LEP combination, and for the search utilising the recoil method, from the OPAL collaboration. We determined normalisation factors for the effective luminosities that we employ 
to approximately account for signal efficiencies and detector effects. We demonstrated that with this input our method reproduces the expected limits from LEP and OPAL for the two types of analyses very well.

After this validation we applied our method for deriving the expected limits for the ILC at $\sqrt{s}=250 \mathrm{GeV}$ for both types of analyses. We used the normalisation factors $c_{\mathrm{bb}}$ and $c_{\text {recoil }}$ that we determined for LEP 2 but assumed as ILC conditions a centre-of-mass energy of $250 \mathrm{GeV}$, beam polarisation of $P_{e^{-}}=-80 \%$ for the electron beam and $P_{e^{+}}=+30 \%$ for the positron beam and used as a very conservative approach the rather modest luminosity of $500 \mathrm{fb}^{-1}$. Our results show that the ILC at $\sqrt{s}=250 \mathrm{GeV}$ will improve the LEP limits in the sensitivity to a light Higgs boson with reduced couplings to gauge bosons for the most interesting mass range between about $60 \mathrm{GeV}$ and $100 \mathrm{GeV}$ by more than an order of magnitude. This sensitivity of the direct search for additional light Higgs bosons at the ILC will go much beyond the indirect sensitivity of the HL-LHC from the rate measurements of the detected state at $125 \mathrm{GeV}$ even for the projected ultimate accuracy reachable at the HL-LHC with $3000 \mathrm{fb}^{-1}$. It is interesting to note in this context that the indirect sensitivity of the ILC with $\sqrt{s}=250 \mathrm{GeV}$ from the rate measurements of the state at $125 \mathrm{GeV}$ is similar to the direct search reach of the ILC via the recoil method, while the direct searches for additional light Higgs bosons at the ILC utilising the $\phi \rightarrow b \bar{b}$ decay will significantly improve even on the ultimate indirect ILC sensitivity.

The physics potential for the direct searches at the ILC with $\sqrt{s}=250 \mathrm{GeV}$ discussed in this paper complements and significantly enhances the ILC physics progmamme for precision measurements in the Higgs and the electroweak sector.

Acknowledgements We thank P. Bechtle, K. Desch, S. Heinemeyer, F. Lika, J. List and A. Raspereza for useful discussions. G.M.P. and G.W. acknowledge support by the Deutsche Forschungsgemeinschaft (DFG, German Research Foundation) under Germany's Excellence Strategy EXC 2121 "Quantum Universe" - 390833306.

Data Availability Statement This manuscript has no associated data or the data will not be deposited. [Authors' comment: There is no additional data. All relevant data is included in the article.]

Open Access This article is licensed under a Creative Commons Attribution 4.0 International License, which permits use, sharing, adaptation, distribution and reproduction in any medium or format, as long as you give appropriate credit to the original author(s) and the source, provide a link to the Creative Commons licence, and indicate if changes were made. The images or other third party material in this article are included in the article's Creative Commons licence, unless indicated otherwise in a credit line to the material. If material is not included in the article's Creative Commons licence and your intended use is not permitted by statutory regulation or exceeds the permitted use, you will need to obtain permission directly from the copyright holder. To view a copy of this licence, visit http://creativecomm ons.org/licenses/by/4.0/.

Funded by SCOAP ${ }^{3}$.

\section{References}

1. R. Barate et al. [ALEPH and DELPHI and L3 and OPAL Collaborations and LEP Working Group for Higgs boson searches], Phys. Lett. B 565, 61 (2003). arXiv:hep-ex/0306033

2. S. Schael et al. [ALEPH and DELPHI and L3 and OPAL Collaborations and LEP Working Group for Higgs Boson Searches], Eur. Phys. J. C 47, 547 (2006). arXiv:hep-ex/0602042

3. G. Abbiendi et al. [OPAL Collaboration], Eur. Phys. J. C 27, 311 (2003). arXiv:hep-ex/0206022

4. T. Aaltonen et al. [CDF Collaboration], Phys. Rev. Lett. 109, 111802 (2012). arXiv:1207.1707 [hep-ex]

5. P. Bechtle, H.E. Haber, S. Heinemeyer, O. Stål, T. Stefaniak, G. Weiglein, L. Zeune, Eur. Phys. J. C 77(2), 67 (2017). arXiv:1608.00638 [hep-ph]

6. E. Bagnaschi et al., Eur. Phys. J. C 79(7), 617 (2019). arXiv: 1808.07542 [hep-ph]

7. F. Domingo, G. Weiglein, JHEP 1604, 095 (2016). arXiv: 1509.07283 [hep-ph]

8. P. Drechsel, L. Galeta, S. Heinemeyer, G. Weiglein, Eur. Phys. J. C 77(1), 42 (2017). arXiv:1601.08100 [hep-ph]

9. F. Domingo, S. Heinemeyer, S. Paßehr, G. Weiglein, Eur. Phys. J. C 78(11), 942 (2018). arXiv:1807.06322 [hep-ph]

10. T. Biekötter, M. Chakraborti, S. Heinemeyer, Eur. Phys. J. C 80(1), 2 (2020). arXiv:1903.11661 [hep-ph]

11. CMS Collaboration [CMS Collaboration], CMS-PAS-HIG-17-013

12. S. Heinemeyer, T. Stefaniak, PoS CHARGED2018, 016 (2019). https://doi.org/10.22323/1.339.0016. arXiv:1812.05864 [hep-ph]

13. [ATLAS Collaboration], ATLAS-CONF-2018-025

14. K. Choi, S.H. Im, K.S. Jeong, C.B. Park, Eur. Phys. J. C 79(11), 956 (2019). https://doi.org/10.1140/epjc/s10052-019-7473-1. arXiv: 1906.03389 [hep-ph]

15. J. Cao, X. Jia, Y. Yue, H. Zhou, P. Zhu, Phys. Rev. D 101(5), 055008 (2020). https://doi.org/10.1103/PhysRevD.101.055008. arXiv:1908.07206 [hep-ph]

16. W.G. Hollik, S. Liebler, G. Moortgat-Pick, S. Paßehr, G. Weiglein, Eur. Phys. J. C 79(1), 75 (2019). https://doi.org/10.1140/ epjc/s10052-019-6561-6. arXiv:1809.07371 [hep-ph]

17. W.G. Hollik, C. Li, G. Moortgat-Pick, S. Paasch, arXiv:2004.14852 [hep-ph]

18. P. Drechsel, G. Moortgat-Pick, G. Weiglein, arXiv:1801.09662 [hep-ph]

19. G. Weiglein, Physics Potential of the ILC at $250 \mathrm{GeV}$. Talk given at the ILC Advisory Panel, MEXT, Tokyo (2018)

20. P. Bock, JHEP 0701, 080 (2007). arXiv:hep-ex/0405072

21. P. Bechtle, SUSY Higgs Boson Searches at LEP and SUSY Parameter Measurements at TESLA. https://doi.org/10.3204/ DESY-THESIS-2004-040

22. W. Kilian, T. Ohl, J. Reuter, Eur. Phys. J. C 71, 1742 (2011). arXiv:0708.4233 [hep-ph]

23. M. Moretti, T. Ohl, J. Reuter, arXiv:hep-ph/0102195

24. R. Assmann, M. Lamont, S. Myers, Nucl. Phys. Proc. Suppl. 109B, 17 (2002)

25. C. Adolphsen et al., arXiv:1306.6328 [physics.acc-ph]

26. K. Fujii et al., arXiv:1710.07621 [hep-ex]

27. J. de Blas et al., JHEP 2001, 139 (2020). arXiv:1905.03764 [hep$\mathrm{ph}]$

28. Y. Wang, [International Large Detector Concept Group], PoS ICHEP2018, 630 (2019)

29. Y. Wang et al. [International Large Detector Concept Group], Talk Given on the International Workshop on Future Linear Colliders (LCWS2018), Arlington, Texas, 22-26 October 2018. arXiv:1902.06118 [hep-ex] 